\title{
Archéopages
}

Archéopages

Archéologie et société

Hors-série 1 | 2008

Construction $^{s}$ de l'archéologie

\section{Au cœur des rapports sociaux : l'imaginaire dont les archéologues ne perçoivent que les restes symboliques}

\section{Maurice Godelier}

\section{(2) OpenEdition}

\section{Journals}

Édition électronique

URL : https://journals.openedition.org/archeopages/859

DOI : 10.4000/archeopages.859

ISSN : 2269-9872

Éditeur

INRAP - Institut national de recherches archéologiques préventives

Édition imprimée

Date de publication : 1 février 2008

Pagination : 77-79

ISSN : 1622-8545

\section{Référence électronique}

Maurice Godelier, «Au cœur des rapports sociaux : I'imaginaire dont les archéologues ne perçoivent que les restes symboliques ", Archéopages [En ligne], Hors-série 1 | 2008, mis en ligne le 01 février 2008, consulté le 26 février 2023. URL : http://journals.openedition.org/archeopages/859; DOI : https://doi.org/10.4000/archeopages.859 
Au cour des rapports sociaux: l'imaginaire dont les archéologues ne perçoivent que les restes symboliques

Maurice Godelier

École des hautes études en sciences sociales

L 'intérêt que je porte à l'archéologie remonte à ma jeunesse, lorsque j'espérais - comme beaucoup - trouver le secret de la genèse de formes nouvelles de sociétés et de modes de pensée. Je m'intéressais particulièrement à la genèse des sociétés à castes et à classes et à l'apparition des premières formes d'État, etc. L'archéologie m'attirait pour des raisons à la fois scientifiques et enfantines, puisque j'en attendais obscurément la résolution d'énigmes, voire de mystères. Il y a d'ailleurs toujours chez moi un peu d'envie lorsque je considère le travail des archéologues. Envie suscitée par l'attrait que leurs découvertes exercent sur un vaste public qui se presse (et paie) pour contempler un casque et quelques ossements. Je me souviens encore d'une place de la petite ville de Cambrai où j'habitais... une excavation à peine protégée $y$ attirait les passants parce qu'une pancarte indiquait: «tombe présumée d'un chef de l'époque des invasions des Wisigoths ». En fait, il n'y avait dans ce trou pratiquement rien à voir, mais les gens étaient fascinés. Les mots et les ossements nourrissaient leur imagination.

Concernant les Wisigoths et les Francs, je devais apprendre par la suite - grâce à la lecture du livre de Georges Duby sur Les Trois Ordres ou l'imaginaire du Moyen Âge - l'importance de la région du Nord dans la genèse du féodalisme. Plus tard, les travaux des archéologues sont venus mettre en doute la thèse de l'importance des invasions germaniques dans la disparition de l'Empire romain. J'ai aussi découvert - grâce à Jean-Paul Demoule que j'avais choisi comme chargé de mission pour l'archéologie, lorsque j'étais directeur du département des sciences de l'homme et de la société au CNRS - que faire revivre des civilisations et des époques disparues coûtait cher; beaucoup plus cher que les enquêtes sur les sociétés vivantes conduites par les ethnologues; un peu moins, cependant, que les modèles mathématiques et statistiques que les économistes exigeaient de vouloir produire pour représenter le système économique dans lequel nous vivions et expliquer ses turbulences aux politiques et au grand public.

De fait, il y eut trois moments et trois contextes, dans ma formation, au cours desquels j'ai lu beaucoup d'archéologie. Ce fut tout d'abord dans le cadre du Groupe de recherches sur l'Antiquité, créé dans les années 1960. Il réunissait Jean-Pierre Vernant, Paul Garelli, Jacques Gernet, Jean Yoyotte, Pierre Vidal-Naquet et quelques autres qui allaient tous faire des carrières scientifiques brillantes. Au cours de nos discussions étaient réanalysées de grandes civilisations : la Mésopotamie, l'Égypte, la Chine et bien sûr la formation des cités grecques.

Une quantité énorme de données archéologiques et autres était au cœur des débats. J'étais le benjamin du groupe et j'appris beaucoup. Je n'étais pas encore entré dans le champ de l'anthropologie.

Quelques années plus tard, ce fut la création, à la Maison des sciences de l'homme, par Jacques Barrau, du Muséum national d'histoire naturelle, et par moi-même, d'un groupe de travail sur les rapports entre «Écologie et Société». Nous étions à l'époque des précurseurs. L'un des thèmes centraux de nos discussions était l'analyse des processus et des conditions historiques de la domestication des plantes et des animaux. Nous nous sommes plongés dans l'archéologie de plusieurs régions du monde où avaient existé les ancêtres sauvages des plantes et des animaux domestiqués par l'homme, et nous nous promenions dans le Néolithique. J'étais en outre intéressé par un problème qui n'avait pas été vraiment abordé par les travaux du groupe de Vernant, celui de la genèse des premières sociétés étatiques, d'où des lectures sur l'Amérique précolombienne, la formation des États ou des cités-États en Orient et Extrême-Orient mais aussi en Afrique et en Europe.

Peu à peu, avec mes années de terrain en Nouvelle-Guinée et un investissement de plus en plus grand dans le domaine de l'anthropologie, mes lectures archéologiques ont diminué, sans disparaître cependant. Cela d'autant qu'au cours des années 1970 il y eut parmi les anthropologues souvent d'inspiration marxiste - de nombreux débats sur l'évolution des formes de société et de pouvoir, et de nombreuses publications traitant d'une hypothétique succession historique et évolutive de trois sortes d'organisation sociale: les tribus, les chefferies et l'État. Je citerai, à cet égard, les écrits de Marshall Sahlins "première manière» (voir son ouvrage Tribesmen publié en 1968), Leslie White, Julian Steward et Laurence Krader. À l'époque, ces collègues étaient influencés par Karl Polanyi ou, sur un autre plan, par les discussions sur le concept marxiste de «mode de production asiatique». Or, ce concept impliquait qu'il aurait existé plusieurs lignes d'évolution des sociétés tribales à chefferies vers des sociétés étatiques: l'une propre à l'Occident depuis l'Antiquité gréco-romaine, les autres différentes et apparues dans l'Orient proche ou lointain, ainsi que dans la Méso-Amérique et l'Amérique du Sud précolombiennes.

Mais revenons à l'archéologie. Ce qui me frappait, dans mes lectures archéologiques, c'était le recours des archéologues - lorsqu'ils ne disposaient pas de sources écrites ou de traditions orales permettant d'interpréter les restes matériels d'une société ou ses signes symboliques à des données accumulées et analysées par les anthropologues sociaux et culturels. C'était comme si, pour redonner vie à des modes de vie et de pensée disparus, les archéologues - 
ne disposant que de l'anatomie matérielle d'un corps social - utilisaient des témoignages ethnologiques pour donner à ce corps

du mouvement et de la vie. Bref... pour en inventer la physiologie. Exercice périlleux et condamné à ne fournir que des images abstraites et floues du passé et, de ce fait, incomplètement crédibles.

Finalement, comparée à la tâche de l'ethnologue, celle de l'archéologue - lorsqu'il ne dispose pas de sources écrites ou orales pour interpréter des vestiges matériels - est difficile. Ce qui risque de lui échapper, en permanence, ce sont les manières qu'avaient les membres d'une société disparue de penser leur univers et leur place dans cet univers. Pour dire les choses de façon plus précise, il est très ardu de reconstituer les contenus imaginaires qui ont donné - pour les acteurs - leur sens aux symboles matériels produits, par ces acteurs, pour transformer ces contenus en réalités visibles (dont certaines sont parvenues jusqu'à nous). Il faut pouvoir - pour comprendre les rites et les pratiques symboliques qui les mettent en ouvre et en actes - reconnaître l'existence et l'importance des dimensions et des contenus imaginaires des institutions sociales.

L'imaginaire et le symbolique sont deux domaines qui, la plupart du temps, sont confondus dans la tête des chercheurs. Il est pourtant fondamental de comprendre que ce sont là deux domaines indissociables, certes, mais de nature distincte.

Le domaine de l'imaginaire est constitué de toutes les manières dont les humains se représentent l'origine et la nature de leur univers, et la place qu'eux-mêmes occupent dans cet univers. L'imaginaire est donc d'abord un monde idéel; un monde qui a sa source dans la pensée. C'est le domaine des représentations. Or, toute représentation étant en même temps une interprétation de ce qu'elle représente, l'imaginaire est l'ensemble des interprétations que l'humanité a pu produire pour se représenter le monde dans lequel elle vit. L'imaginaire est donc un monde socialement réel, mais d'abord composé de réalités mentales. Cependant, il est évident que si ces réalités idéelles restaient uniquement à l'intérieur de la tête des individus qui les produisent, elles resteraient inconnues des membres de la société à laquelle ils appartiennent et n'auraient pas ou peu d'efficacité au sein de cette société et sur elle. En revanche, tout change dès que ces idéalités sont communiquées et partagées par un grand nombre. C'est là qu'entre en scène le domaine des symboles et des pratiques symboliques.

Le domaine du symbolique est précisément l'ensemble des moyens par lesquels des réalités mentales et idéelles revêtent des formes et s'incorporent dans des matières qui les rendent communicables. Ces formes matérielles peuvent être très diverses. Ce sont des monuments, temples et palais, des postures du corps (génuflexion, par exemple) ou des parures réservées à certains pour témoigner de leur statut et de leur fonction, et qui les accompagnent jusque dans leur tombe. Et bien entendu, ce sont aussi des mots et des sons, hymnes adressés aux dieux, danses et musiques, récits mythiques, etc.

Toutes ces réalités symboliques sont donc fondamentalement du langage. Mais un langage qui déborde les langues parlées qui, pour beaucoup d'entre elles, ont aujourd'hui disparu. Le problème est donc le suivant: l'imaginaire joue un rôle essentiel dans la vie sociale mais ne peut acquérir d'existence et d'efficacité sociales sans s'incarner dans des signes et des pratiques symboliques, des rites, des monuments, etc. Mais le symbolique n'est pas seulement destiné à communiquer des contenus de pensée qui donnent sens à des formes d'organisation sociale. Il sert également à prouver aux membres d'une société la «vérité» de ces contenus imaginaires car il les transforme en des réalités qui tombent sous leurs sens et donnent lieu à des séquences d'actes (les rites, par exemple) qui «démontrent» que les dieux invoqués existent. Un rite implique toujours des individus et des groupes qui, à travers ces rites, entrent dans des rapports spécifiques avec des ancêtres, des dieux, etc. Les pratiques symboliques font donc naître et constituent un ensemble de rapports sociaux originaux entre les individus et entre les groupes qui composent une société, et ne sont pas seulement un ensemble de signes dont les acteurs comprennent le sens.

Je comprends la difficulté du métier de l'archéologue lorsque, découvrant les ruines d'un temple, d'un palais, des peintures murales ou d'autres signes, il s'efforce de reconstruire le sens que tout cela a pu avoir pour les individus et les groupes qui vivaient dans la société qui les a produits. Difficile également de trouver les raisons de la transformation de ces signes et de ces restes s'il constate que ceux-ci ont évolué ou disparu au cours des siècles. Il est pourtant aujourd'hui amplement démontré que les archéologues savent «faire des miracles» et sont passés d'une archéologie de la découverte d'objets spectaculaires à une archéologie minutieuse, statistique et rigoureuse qui ne néglige aucun indice (matériel) pour interpréter le passé.

Je voudrais également insister sur le fait queau cœur de toutes les sociétés - les rapports que nous appelons, en Occident, "politico-religieux» occupent une place stratégique. Les archéologues nous en fournissent d'innombrables preuves puisque, parmi les sites qu'ils explorent, beaucoup sont des tombes de chefs, des plateformes de temples, etc. À côté de ces temples, on trouve souvent des fossés avec, par exemple, des restes de sacrifices offerts à des dieux. Il est d'ailleurs intéressant de constater que les données archéologiques fournissent beaucoup plus d'informations sur la nature des rapports politicoreligieux que sur la nature des rapports de parenté qui pouvaient exister dans une société disparue. Je me souviens de textes déconcertants d'archéologues essayant de prouver l'existence 
de rapports de parenté matrilinéaires au sein d'une société disparue à partir de la présence de traces de céréales incrustées dans des poteries qu'on supposait avoir été fabriquées par les femmes. Le raisonnement était difficile à suivre et les preuves inexistantes.

Enfin, je voudrais ajouter que les archéologues ont augmenté notre connaissance en nous faisant découvrir de multiples sites au sein desquels des sociétés célébraient leurs dieux ou communiquaient avec les esprits de la nature qui les entourait. L'existence et la multiplicité de ces sites posent la question des rapports changeants entre le divin et l'humain, ainsi que le problème de l'importance des rites pour agir sur la nature et pour établir et conserver un ordre social. Or l'une de nos grandes difficultés, tant pour les archéologues que pour les ethnologues occidentaux, est de comprendre la rupture qu'a représentée l'apparition, au Proche-Orient, de religions monothéistes. La plupart des sociétés humaines ont été, et pour beaucoup d'entre elles continuent d'être, polythéistes. Or le rapport au divin et au sacré est totalement différent pour ceux qui croient en une religion polythéiste. Les hommes ont alors le sentiment d'accomplir les rites avec leurs dieux et leurs ancêtres. Ceux-ci sont présents à leur côté lorsqu'ils célèbrent les rites ou se rendent visibles en s'incarnant, par exemple, provisoirement dans le masque des danseurs.

N'oublions pas que - pour évoquer ici l'Europe préchrétienne-, dans la Rome antique, toutes les grandes cérémonies comportaient des sacrifices. Ces sacrifices se concluaient par un banquet où les dieux des Romains étaient assis aux côtés des magistrats et des prêtres qui avaient célébré les rites. La distance entre l'humain et le divin était donc totalement différente de celle introduite par le monothéisme. L'idée qu'il n'existe qu'un seul Dieu - qui a tout créé à partir de rien implique et impose l'idée d'une distance infinie entre les hommes et Dieu, d'une transcendance divine irréductible. De ce fait, les hommes ne peuvent vivre leurs rapports à Dieu que dans un état de subordination absolue. Leur destin dépend de sa grâce et, de ce fait, logiquement de l'amour que Dieu peut avoir pour les hommes. Or aimer les hommes n'était pas une caractéristique obligée ni attendue des dieux des religions polythéistes: les sacrifices qu'on leur rendait n'avaient pas le même sens que les prières et les rites des religions monothéistes. Quelles traces ont donc laissé pour nos générations et pour les archéologues qui les analysent aujourd'hui tous ces domaines de la vie sociale et toutes les différences inventées par les hommes dans la manière d'organiser cette vie et de se représenter l'univers? Une conclusion s'impose: archéologues, ethnologues, historiens ne peuvent que travailler ensemble pour reconstituer avec un peu de «vrai-semblance » quelques morceaux de notre histoire. Aucune de ces disciplines n'y suffira jamais à elle seule, et c'est mieux de le savoir.

\section{L'ethnoarchéologie: de la ferme de La Tour au tell de Houlouf, en passant par un rêve brésilien}

\author{
Augustin F. C. Holl \\ Museum of Anthropology, University of Michigan
}

J uin 1980 : dans la cour de la ferme de La Tour à Paissy, dans l'Aisne, la campagne de fouille archéologique du Centre de recherches protohistoriques de l'université Paris I bat son plein. Cette année-là, je participais pour la première fois à une fouille. Il va sans dire que mes compétences étaient abstraites. J'ai été adopté par l'équipe de la vallée de l'Aisne, et c'est elle qui m’a formé.

Au fil du temps, je me suis intéressé sérieusement aux recherches sur les premières communautés agricoles du Bassin parisien. Les sites de Cuiry-lès-Chaudardes et Berry-au-Bac révélaient des groupes de longues maisons, caractéristiques de ces communautés pionnières [Fig.1]. Les membres de l'équipe de la vallée de l'Aisne, ainsi que d'autres chercheurs allemands, belges, néerlandais, polonais, autrichiens, tchécoslovaques, etc., s'attelaient à l'étude des longues maisons néolithiques dans toutes leurs manifestations archéologiques. Chaque maison avait abrité des individus faisant partie d'un groupe social qui se distinguait des autres, tout en formant entre elles une entité sociale globale, robuste, flexible et durable. Quelle était l'éventuelle structure de cette entité sociale? Comment se reproduisait-elle? Comment s'ajustait-elle aux changements démographiques et économiques? Il est difficile, pour l'archéologue, de répondre à ces questions et la tentation était très forte de ne même pas les prendre en considération. Les données archéologiques ne permettaient au mieux que de faire des suggestions. Que faire?

Eurêka! Par la suite et au cours des multiples conversations professionnelles et amicales que j'ai pu avoir durant de mes années parisiennes, j'ai eu l'idée d'une recherche ethnoarchéologique sur la dynamique des maisons longues. Il fallait trouver une région du monde hébergeant encore des sociétés utilisant ce type de maison. Je m'en suis ouvert à un collègue ethnologue nanterrois qui avait alors un projet de recherche au Brésil: il y avait effectivement des sociétés à maisons longues le long du rio Xingu, un important affluent de l'Amazone. Ne pouvant plus aller sur ce terrainlà, il me proposa de me mettre en contact avec un ethnologue de l'Orstom qui travaillait dans cette région du monde, au cours des années 1980. L'éventualité d'un projet ethnoarchéologique en Amazonie m'a alors fait perdre tout sens de la mesure: j'ai oublié que j'avais des cours à donner, un projet archéologique au Nord Cameroun et un autre dans le Néguev en Israël. Le rêve s'est heurté à la dure réalité, 